
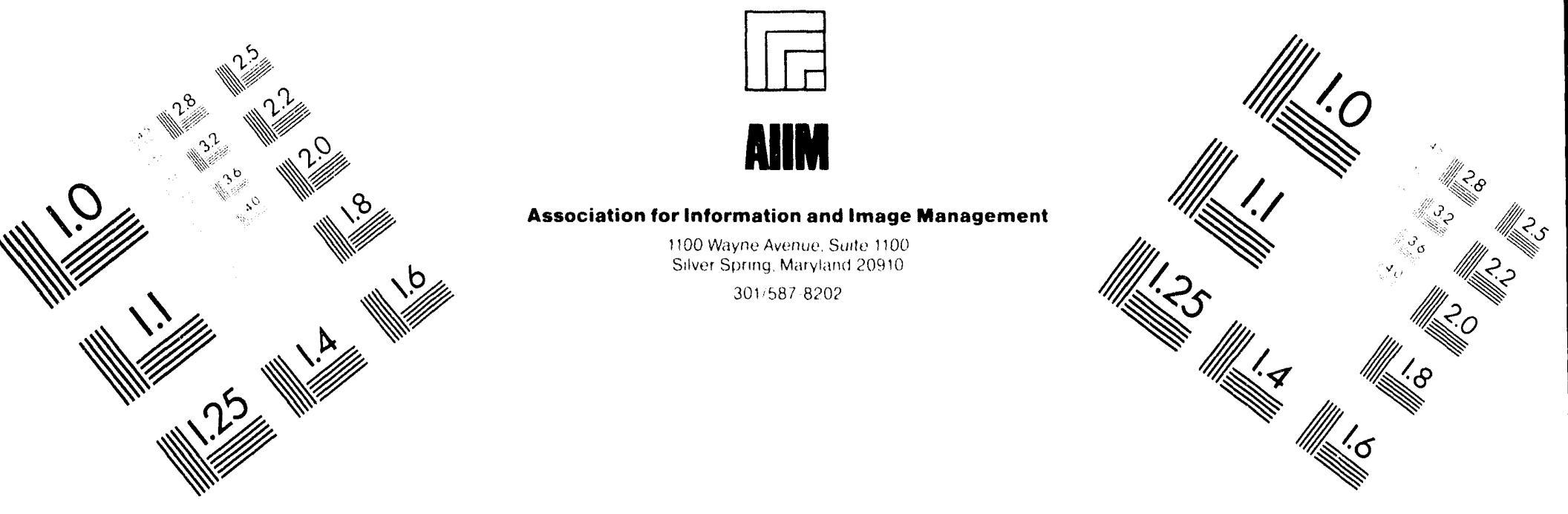

\title{
Centimeter
}

$\begin{array}{llllllllllllllll}1 & 2 & 3 & 4 & 5 & 6 & 7 & 8 & 9 & 10 & 11 & 12 & 13 & 14 & 15 & \mathrm{~mm}\end{array}$ huludm

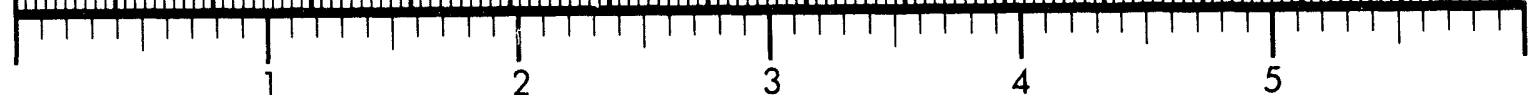
Inches
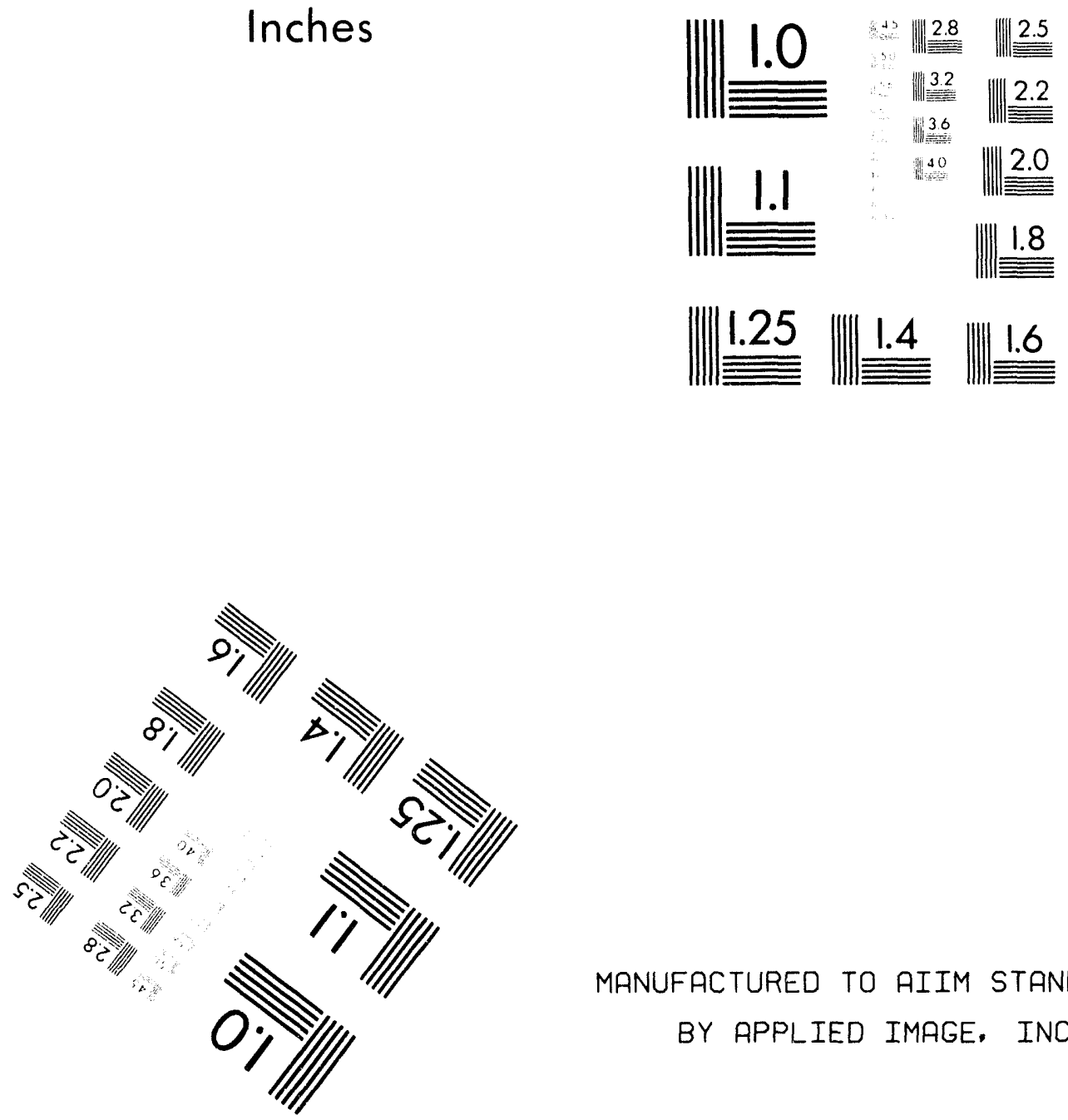

MANUFACTURED TO AIIM STANDARDS BY APPLIED IMAGE, INC.

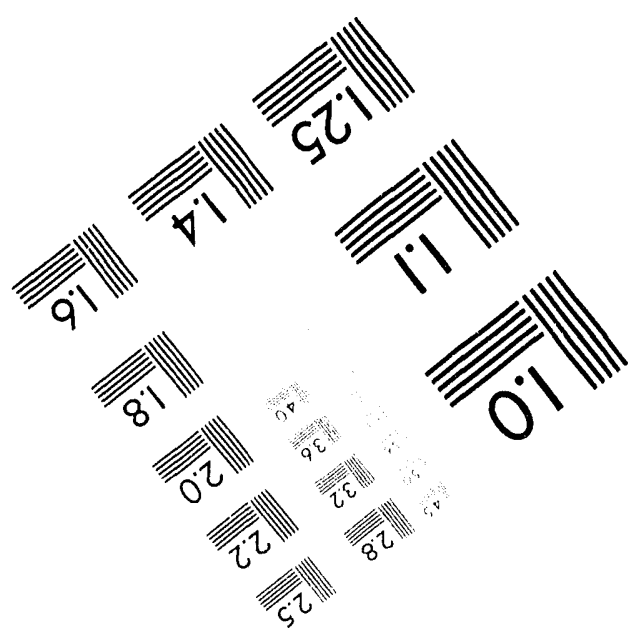





\title{
$\log _{-1}-9405 / 36-8$
}

Submitted to the

1994 Joint USA-Russia Energetic Material Technology Symposium

Livermore, Californio (May 18-25, 1994)

EFFECTS OF TWO-PHASE FLOW ON THE

DEFLAGRATION OF POROUS ENERGETIC MATERIALS

\author{
Stephen B. Margolis" and Forman A. Williams** \\ -Combustion Research Facility \\ Sandia National Laboratories ${ }^{\dagger}$ \\ Livermore, Californis 94551-0969 USA \\ " Department of Applied Mechanics and Engineering Sciences \\ University of California, San Diego \\ La Jolla, California 92093-0310 USA
}

\section{PRESENTATION ABSTRACT}

The combustion behavior of energetic materials (e.g., solid propellants) has long been of interest in the fields of propulsion and pyrotechnics. In many such applications, it is becoming increasingly clear that two-phase flow effects play an important role, especially since, during combustion, most homogeneous solid propellants develop thin multi-phase layers at their surfaces in which finite-rate exothermic reactions occur. In addition, there is a growing interest in the behavior of porous energetic solids, since even initially dense materials can develop significant void fractions if, at any time, they are exposed to abnormal thermal environments. The deflagration characteristics of such "damaged" materials may then differ significantly from those of the pristine material due, at least in part, to gas flow in the solid/gas preheat region. The presence of gas in the porous solid in turn results in a more pronounced two-phase effect in the multi-phase surface layer, such as in the liquid melt region of nitramine propellants, which thus tend to exhibit extensive bubbling in an exothermic foam layer. The present analysis is largely applicable to this latter class of propellants.

Describing phenomena associated with two-phase flow is inherently much more difficult than analyzing those occurring within a single phase. There are, first of all, certain fundamental difficulties that generally require the formulation of constitutive relations in order to obtain a closed model (cf. Drew [1], Baer and Nunziato [2]). Second, but of even greater significance from the standpoint of analysis, is the fact that the degree of nonlinearity in any model is increased by the appearance of appropriate volume-fraction variables that multi. ply each quantity associated with a particular phase. As a consequence, much of the early two-phase work in this area tended to treat the two-phase medium as a single phase with suitably "averaged" properties (cf. Maksimov and Merzhanov [3], Merzhanov (4]). This, in effect, requires the velocity (and temperature) of each phase to be same, precluding many of the predominant effects associated with combustion processes that involve two-phase flow.

\footnotetext{
- Presenter

† This work was performed at Sandia National Laboratories supported by the U.S. Department of Energy under contract DE-AC04-94AL85000
}

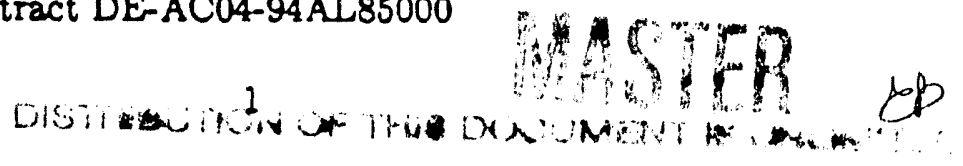


We have recently addressed some of these concerns in several papers (Margolis, Williams and Armstrong [5], Margolis and Williams [6], Li, Williams and Margolis [7]). To be able to focus clearly on the effects of two-phase flow, the description of the chemistry was deliberately simplified. In particular, a one-step exothermic process

$$
R(c) \rightarrow P(g)
$$

was considered in [5] and [6], where $R(c)$ is the condensed (liquid) reactant, and $P(g)$ is the gaseous product. Thus, each phase is a pure species, and gas-phase reactions were either neglected or assumed to be remote, as was the solid/liquid interface. In (7), a more elaborate mechanism, motivated by knowledge of nitramine chemistry and given by

$$
R(c) \rightarrow P(g), \quad R(c) \leftrightarrow R(g), \quad R(g) \rightarrow P(g)
$$

was adopted, where $R(g)$ is a gaseous reactant. In each of these studies, the goal was to clarify certain two-phase effects associated with different velocities (and, in [5] and [6], temperatures) for each phase. Though not directly applicable to propellant deflagration, related two-phase modelling that accounts for velocity differences between phases has been used in the study of filtration combustion (cf. Aldushin [8], Shkadinsky et al. [9]).

The purpose of the present work is to extend the analysis of [5] in several important respects. First, we formulate a more complete problem that explicitly includes melting of the unburned solid. Second, we assume that the unburned solid material has nonzero porosity, resulting in two-phase flow throughout the preheat and reaction zones. In our previous work, the effects of two-phase flow were confined to the reaction zone, which would be equivalent to assuming zero porosity for the solid in the present model. Finally, the fact that the gas phase exists throughout the unburned solid/liquid material leads us to relax the constant-density assumption that was adopted for the gas in our earlier work. In particular, we now allow for variable gas density according to a gas-phase equation of state that allows us to consider the effects of pressure on the various quantities of interest. These generalizations lead to a number of interesting effects directly attributable to the influences of two-phase flow. For the present, we confine our attention to steady deflagration, leaving consideration of instability and other nonsteady effects (cf. [6]) for future work.

Some of the results that have been obtained thus far are as follows. First, a multiphase flow theory has been developed for the deflagration of porous energetic materials, such as degraded nitramine propellants, that undergo exothermic reactions in a liquid layer to produce gaseous products. Second, both single- and two-temperature models have been analyzed, the latter in a perturbative fashion for large, but finite, interphase heat-transfer coefficients. The combination of porosity and gas-phase thermal expansion has been shown to lead to pressure-dependent temperatures, resulting in a significant pressure sensitivity for the burned temperature, and hence the propagation speed. Formulas for the latter have been derived for the case of steady, planar burning using the method of activation-energy asymptotics. These formulas demonstrate that increases in the conductivity of either phase in the liquid/gas reaction region lead to increases in the burning velocity, while increased resistance to interphase heat transfer generally has a similar effect due to the tendency of the condensed phase, where the beat of reaction is initially deposited, to have a higher temperature than that of the gas. 
Sandia National Laboratories

\section{EFFECTS OF TWO-PHASE FLOW ON THE} DEFLAGRATION OF POROUS ENERGETIC MATERIALS

Stephen B. Margolis ${ }^{\dagger}$ and Forman A. Williams ${ }^{\ddagger}$

${ }^{\dagger}$ Combustion Research Facility

Sandia National Laboratories

Livermore, California 94551-0969

$\ddagger$ Department of Applied Mechanics and Engineering Sciences

University of California, San Diego

La Jolla, Cailfornia 92093-0310

\section{Overall Program:}

Model Post-Ignition Combustion Behavior and Burn Dynamics of Porous Energetic Solids

1. Predict detailed deflagration structure, propagation velocity, etc.

2. Analyze stability of combustion wave and its nonlinear evolution

3. Determine likelihood of uncontrolled burn (transition to detonation) 
PHYSICAL MODEL

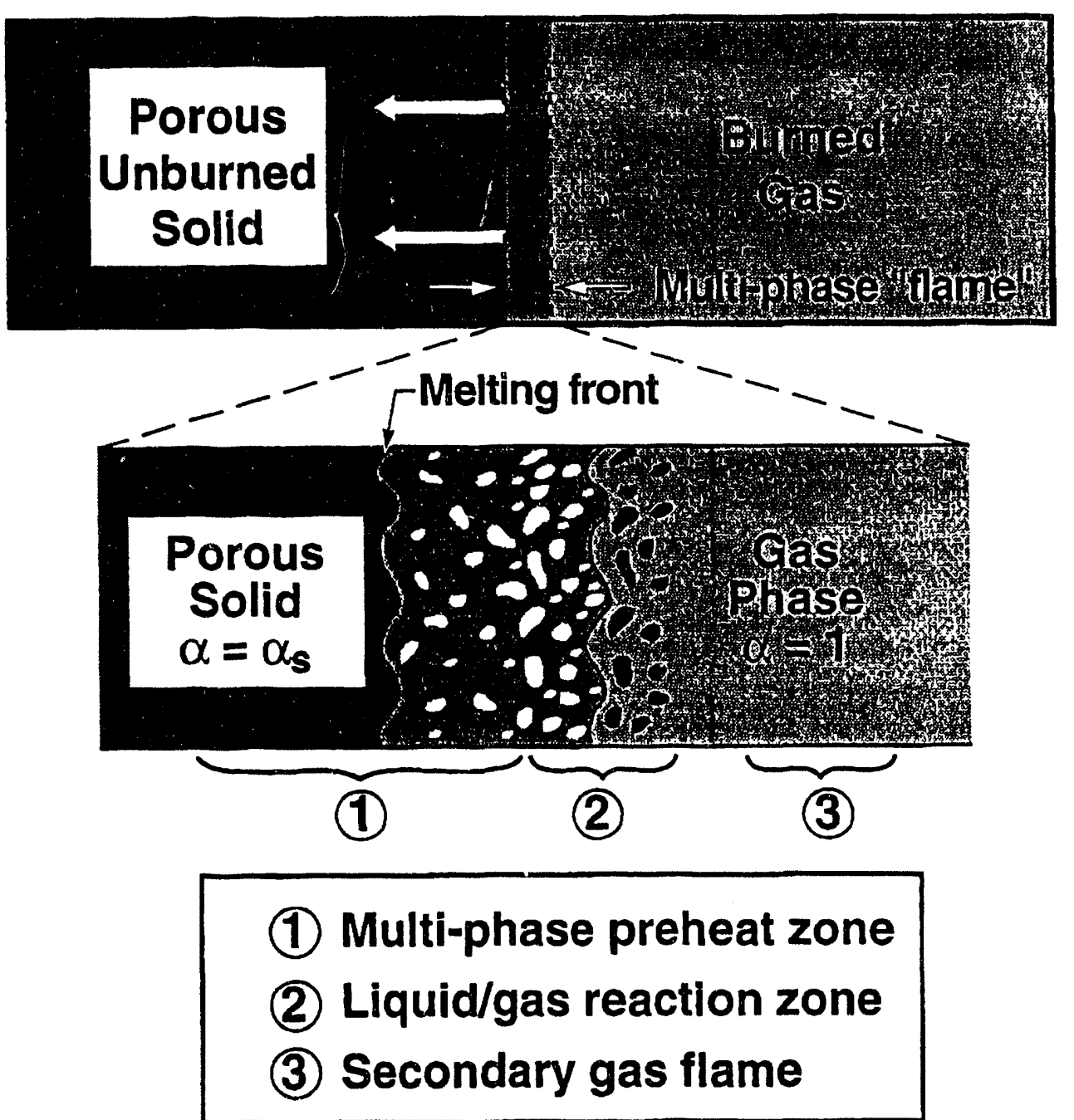

Important Effects:

1. Multi-phase flow (solid/gas, liquid/gas)

2. Porosity of unburned (degraded) material

3. Boundary conditions (unconfined/confised)

4. Reaction rate dependencies on pressure, temperature 
Recent Results/Efforts:

1. Development of mathematical models for unconfined/confined multiphase deflagration of porous energetic solids

2. Determination of basic asymptotic solutions for steadily-propagating, unconfined deflagration waves

3. Stability analyses of unconfined deflagration for nonporous materials; prediction of oscillatory burning

Future Results/Efforts:

1. Predict stability of deflagration structure for porous energetic materials in both unconfined and confined geometries (linear, cylindrical, spherical)

2. Analyze time evolution of confined deflagration; predict onset of transition to detonation

3. Determine likelihood of controlled versus uncontrolled burn as function of model parameters

4. Correlate results with LLNL/HEAF strand-burner experiments 
MATHEMATICAL MODELS FOR MULTI-PHASE REACTING FLOW

Global Reaction Schemes for Nitramine-Type Propellants:

$$
R(c) \rightarrow P(g) \text { and } R(c) \leftrightarrow P(g), R(c) \leftrightarrow R(g), R(g) \rightarrow P(g)
$$

Early work: single-temperature, single-velocity models

Maksimov and Merzhanov (1966), Merzhanov (1969)

General models for multi-phase flow:

Drew (1983), Baer and Nunziato (1986)

Present work: Different velocities, single- and two-temperature models

Margolis, Williams and Armstrong (1987), Margolis and Williams (1990)

Li, Williams and Margolis (1990) (zero porosity, constant gas density)

Margolis and Williams (1994) (nonzero porosity, variable gas density) 
I. Steady 1-D Deflagration of Unconfined Porous Material

Nondimensional variables and parameters:

$\alpha$ Gas-phase volume fraction

$u_{l, g} \quad$ Liquid- and gas-phase velocities

$p_{g} \quad$ Gas Pressure

$T_{s, l, g} \quad$ Solid, liquid, gas-phase temperatures

$r, \hat{r} \quad$ Liquid-to-solid, (ambient) gas-to-solid density ratios

$b, \hat{b} \quad$ Liquid-to-solid, gas-to-solid heat capacity ratios

$l, \hat{l} \quad$ Liquid-to-solid, gas-to-solid thermal conductivity ratios

$K_{s g}, K_{l g} \quad$ Interphase heat-transfer coefficients

$Q, \gamma_{s}$ heat release, heat of melting

$\Lambda \quad$ Burning-rate eigenvalue

1. Overall continuity:
a. $\quad \alpha_{s} \frac{d}{d \xi}\left[\rho_{g}\left(u_{g}+1\right)\right], \quad \xi<0$
b. $\frac{d}{d \xi}\left[r(1-\alpha)\left(u_{l}+1\right)+\hat{r} \alpha \rho_{g}\left(u_{g}+1\right)\right], \quad \xi>0$

2. Condensed continuity:
a. $u_{s}=0, \quad \xi<0$
b. $\frac{d}{d \xi}\left[(1-\alpha)\left(u_{l}+1\right)\right]=-\Lambda(1-\alpha) \exp \left[N\left(1-T_{b} / T_{l}\right)\right], \quad \xi>0$ 
3. Condensed energy (using condensed continuity):
a. $\frac{d T_{s}}{d \xi}=\frac{d^{2} T_{s}}{d \xi^{2}}+K_{s g}\left(T_{g}-T_{s}\right), \quad \xi<0$
b. $\quad r(Q+b) \frac{d}{d \xi}\left[(1-\alpha)\left(u_{l}+1\right) T_{l}\right]$

$$
=l \frac{d}{d \xi}\left[(1-\alpha) \frac{d T_{l}}{d \xi}\right]+r b K_{l g}\left(T_{g}-T_{l}\right), \quad \xi>0
$$

4. Overall energy (using condensed continuity):

a. $\left(1-\alpha_{s}\right) \frac{d T_{s}}{d \xi}+\hat{r} \hat{b} p_{g}^{0} \alpha_{s} \frac{d}{d \xi}\left(u_{g}+1\right)$

$$
=\frac{d}{d \xi}\left[\left(1-\alpha_{s}\right) \frac{d T_{s}}{d \xi}+\hat{l} \alpha_{s} \frac{d T_{g}}{d \xi}\right], \quad \xi<0
$$

b. $\frac{d}{d \xi}\left[r(1-\alpha)\left(u_{l}+1\right)\left(Q+b T_{l}\right)+\hat{r} \hat{b} p_{g}^{0} \alpha\left(u_{g}+1\right)\right]$

$$
=l \frac{d}{d \xi}\left[(1-\alpha) \frac{d T_{l}}{d \xi}+\frac{\hat{l}}{l} \alpha \frac{d T_{g}}{d \xi}\right], \quad \xi>0
$$


5. Equation of State: $\rho_{g} T_{g}=p_{g}^{0}+\mathrm{O}\left(M^{2}\right)$ or $\rho_{g}=$ const.

6. Momentum $\Rightarrow$ constitutive relation: $u_{l}=\frac{1}{r}(1-r-s \alpha)$,

where $s$ is a velocity-perturbation parameter determined by

a. viscous effects (increase $\tilde{u}_{l}$, decrease $\tilde{u}_{g}$ )

b. surface-tension gradient effects (decrease $\tilde{u}_{l}$, increase $\tilde{u}_{g}$ )

Marangoni effects $\gg$ viscous effects $\Rightarrow s \geq 0$

7. Continuity, flux conservation across melting surface $\xi=0$ :

a. $T_{s}=T_{l}=T_{m}, T_{g}$ continuous

b. $\alpha^{+} u_{g}^{+}-\alpha_{s} u_{g}^{-}=\alpha_{s}-\alpha^{+}=s \alpha^{+}\left(1-\alpha^{+}\right)$

c. $\alpha^{+} d T_{g} /\left.d \xi\right|_{0^{+}}-\alpha_{s} d T_{g} /\left.d \xi\right|_{0^{-}}=0$

d. $l\left(1-\alpha^{+}\right) d T_{l} /\left.d \xi\right|_{0^{+}}-\left(1-\alpha_{s}\right) d T_{s} /\left.d \xi\right|_{0^{-}}$ $=\left(1-\alpha_{s}\right)\left|\gamma_{s}\right|+\left[b\left(1-\alpha^{+}\right)\left(1-s \alpha^{+}\right)-\left(1-\alpha_{s}\right)\right] T_{m}$

8. Boundary conditions:

a. $\alpha=\alpha_{s}, \quad \xi<0$

b. $u_{g} \rightarrow 0, T_{g} \rightarrow T_{s} \rightarrow 1$ as $\xi \rightarrow-\infty$

c. $\alpha \rightarrow 1, T_{l} \rightarrow T_{g} \rightarrow T_{b}$ (unknown) as $\xi \rightarrow+\infty$

Solution by asymptotic methods for large activation energy (exploit largeness of Zel'dovich number $\beta=\left(1-T_{b}^{-1} \gg 1\right)$

$\Rightarrow$ reaction zone becomes a thin front located at $\xi=\xi_{r}$

$$
\Rightarrow \tilde{U}^{-2} \propto \Lambda \sim \beta\left(\Lambda_{0}+\beta^{-1} \Lambda_{1}+\cdots\right)
$$


Asymptotic Approach

Consider Arrhenius factor $\tilde{A} \exp \left(-\tilde{E}_{l} / \tilde{R}^{\circ} \tilde{T}\right)$ :

$\tilde{E}_{l} / \tilde{R}^{\circ} T_{b} \gg 1 \Rightarrow$ rewrite reaction term as

$$
\tilde{A} \exp \left(-\frac{\tilde{E}_{l}}{\tilde{R}^{\circ} \tilde{T}}\right)=\tilde{\Lambda} \exp \left[-\frac{\tilde{E}_{l}}{\tilde{R}^{\circ} \tilde{T}_{b}}\left(\frac{\tilde{T}_{b}}{\tilde{T}}-1\right)\right]
$$

where

$$
\tilde{\Lambda}=\tilde{A} \exp \left(-\frac{\tilde{E}_{l}}{\tilde{R}^{\circ} \tilde{T}_{b}}\right)
$$

Result: $N \equiv \tilde{E}_{l} / \tilde{R}^{\circ} T_{b} \gg 1 \Rightarrow$

reaction rate exponentially small unless $T \sim T_{b}-\mathrm{O}\left(N^{-1}\right)$

$\Rightarrow$ thin two-phase reaction zone following reactionless multiphase preheat zone

$\Rightarrow$ Tractable solution in each region, matched asymptotically 
Solution to Single-Temperature Model $\left(K_{s g}, K_{l g} \rightarrow \infty\right)$ :

Outer Solution:

$$
\begin{gathered}
\alpha= \begin{cases}\alpha_{s}, & \xi<\xi_{r} \\
1, & \xi>\xi_{r}\end{cases} \\
u_{g}= \begin{cases}T-1, \\
\hat{r}^{-1}\left(1-\alpha_{s}+\alpha_{s} \hat{r}\right) T_{b}-1 \equiv u_{g, \infty}, & \xi>\xi_{r}\end{cases} \\
T(\xi)= \begin{cases}1+\left(T_{m}^{\prime}-1\right) \exp \left[\frac{1+\alpha_{s}(\hat{r} \hat{b}-1)}{1+\alpha_{s}(\hat{l}-1)} \xi\right], & \xi<0 \\
B+\left(T_{m}-B\right) \exp \left[\frac{b\left(1-\alpha_{s}\right)+\hat{r} \hat{b} \alpha_{s}}{l\left(1-\alpha_{s}\right)+\hat{l} \alpha_{s}} \xi\right], & 0<\xi<\xi_{r} \\
T_{b}, & \xi>\xi_{r},\end{cases}
\end{gathered}
$$

where

$$
\begin{gathered}
B \equiv \frac{\left(1-\alpha_{s}\right)\left(1-\left|\gamma_{s}\right|\right)+\hat{r} \hat{b} \alpha_{s}}{b\left(1-\alpha_{s}\right)+\hat{r} \hat{b} \alpha_{s}} \\
\xi_{r}=\frac{l\left(1-\alpha_{s}\right)+\hat{l} \alpha_{s}}{b\left(1-\alpha_{s}\right)+\hat{r} \hat{b} \alpha_{s}} \ln \left(\frac{T_{b}-B}{T_{m}-B}\right) \\
T_{b}=\frac{\left(1-\alpha_{s}\right)\left(Q+1-\left|\gamma_{s}\right|\right)+\hat{r} \hat{b} \alpha_{s}}{\hat{b}\left[1+\alpha_{s}(\hat{r}-1)\right]}
\end{gathered}
$$


Burned Temperature: $T_{b}=\frac{\left(1-\alpha_{s}\right)\left(Q+1-\left|\gamma_{s}\right|\right)+\hat{r} \hat{b} \alpha_{s}}{\hat{b}\left[1+\alpha_{s}(\hat{r}-1)\right]}$

$$
\text { where } \hat{r}=\frac{\tilde{\rho}_{g}^{u}}{\tilde{\rho}_{s}}=\frac{\tilde{p}_{g}^{\circ}}{\tilde{\rho}_{s} \tilde{c}_{g}\left(1-\gamma^{-1}\right) \tilde{T}_{u}}
$$

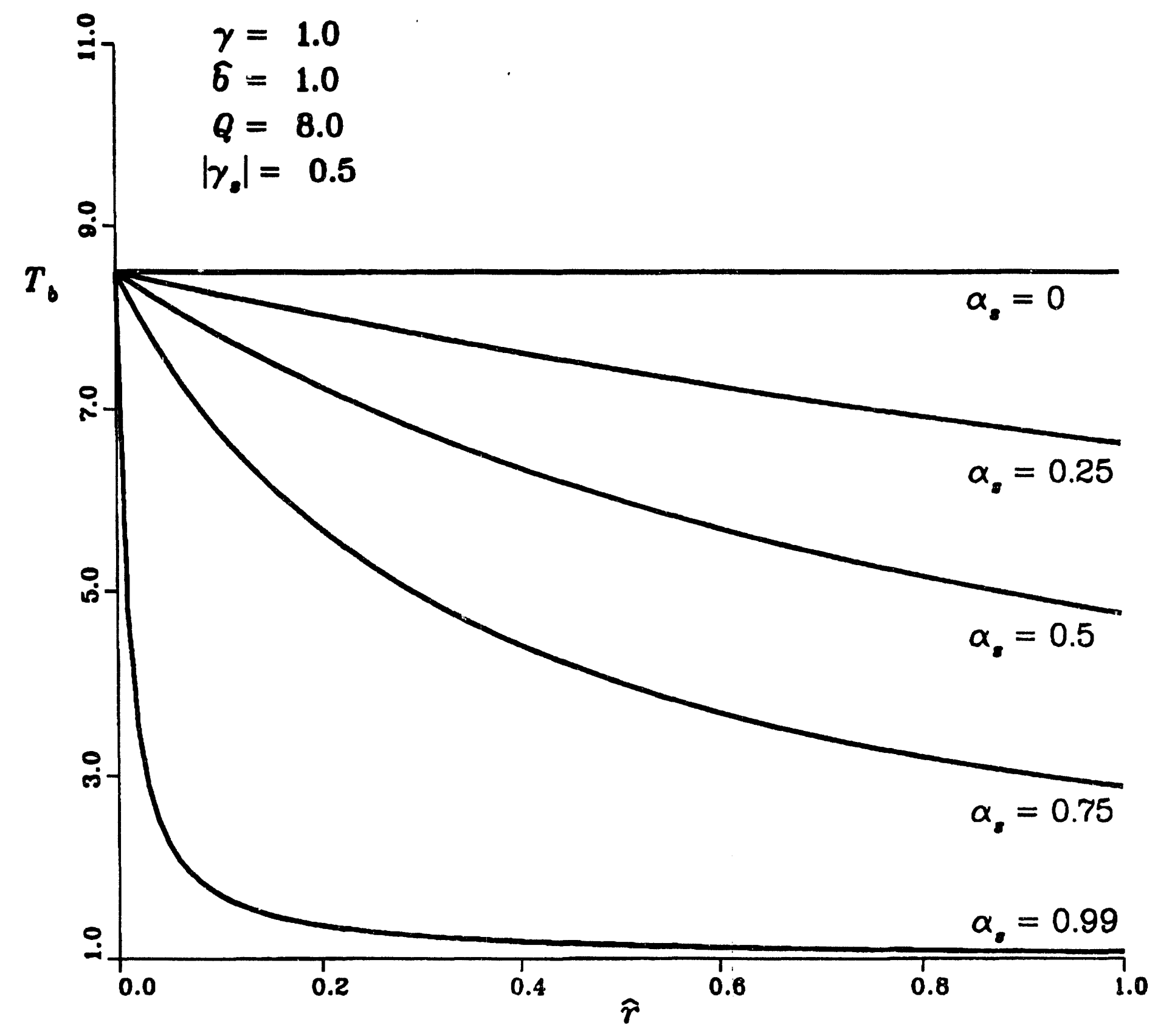


Final Gas Velocity: $u_{g, \infty}=\frac{1+\alpha_{s}(\hat{r}-1)}{\hat{r}} T_{b}-1$

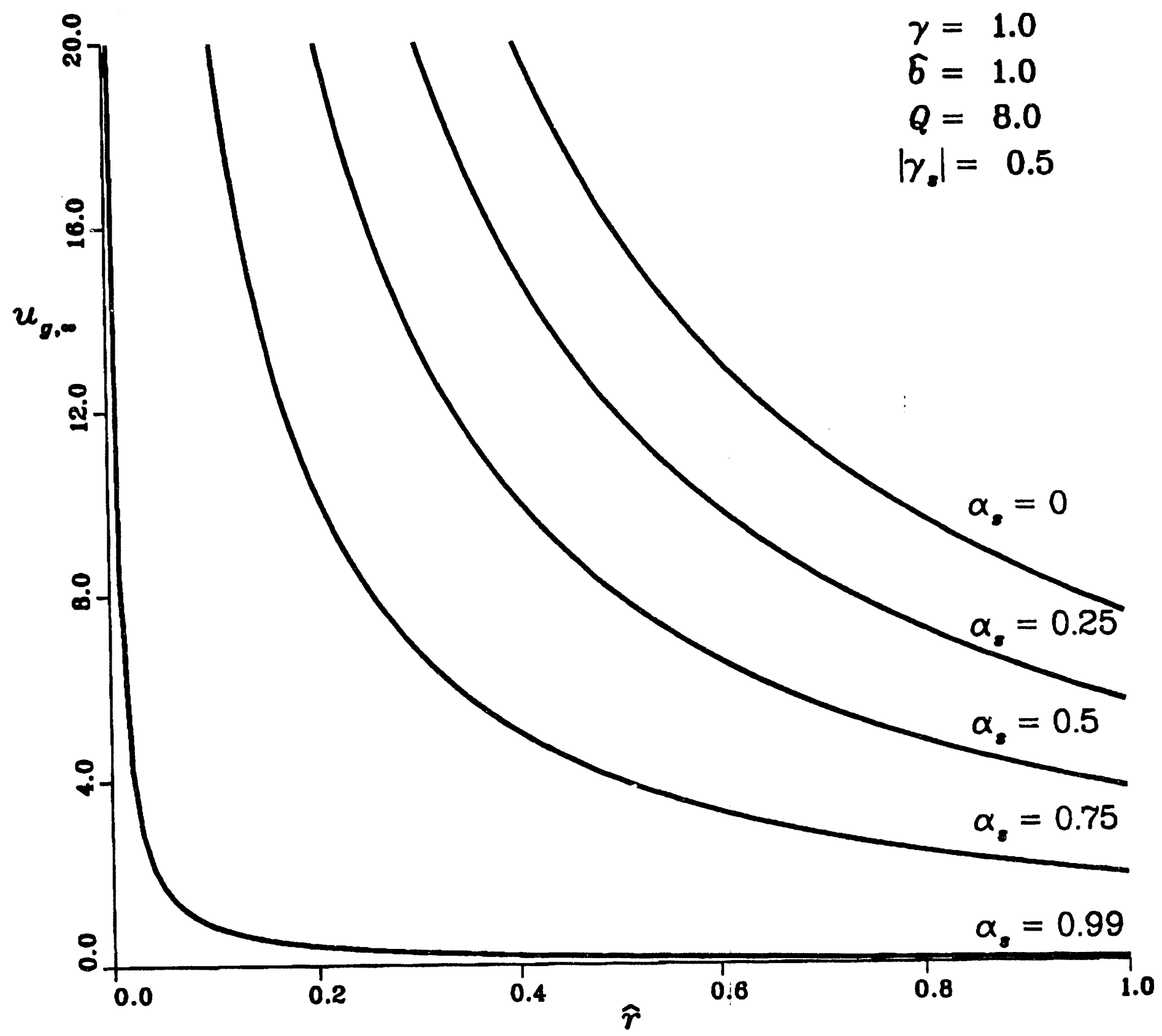


Inner (Reaction-Zone) Solution

$$
\Theta=\frac{T-1}{T_{b}-1}, \quad \eta=\beta\left(\xi-\xi_{r}\right), \quad \beta \equiv\left(1-T_{b}^{-1}\right) N \gg 1
$$

Seek solutions as

$$
\begin{aligned}
\alpha & \sim \alpha_{0}+\beta^{-1} \alpha_{1}+\beta^{-2} \alpha_{2}+\cdots, \\
u_{g} & \sim u_{0}+\beta^{-1} u_{1}+\beta^{-2} u_{2}+\cdots, \\
\Theta & \sim 1+\beta^{-1} \theta_{1}+\beta^{-2} \theta_{2}+\cdots, \\
\Lambda & \sim \beta\left(\Lambda_{0}+\beta^{-1} \Lambda_{1}+\beta^{-2} \Lambda_{2}+\cdots\right) . \\
\Rightarrow \quad \frac{d \alpha_{0}}{d \eta}=r \Lambda_{0}\left(1-\alpha_{0}\right) e^{\theta_{1}} & \\
& {\left[l+(\hat{l}-l) \alpha_{0}\right] \frac{d \theta_{1}}{d \eta}=\frac{D}{T_{b}-1}\left(1-\alpha_{0}\right) }
\end{aligned}
$$

Matching conditions:

$$
\begin{gathered}
\alpha_{0} \rightarrow \alpha_{s}, \quad \theta_{1} \sim E \eta \text { as } \eta \rightarrow-\infty \\
\alpha_{0} \rightarrow 1, \quad \theta_{1} \rightarrow 0 \text { as } \eta \rightarrow+\infty
\end{gathered}
$$

where

$$
D \equiv(b-\hat{b}) T_{b}+Q,\left.\quad E \equiv \frac{1}{T_{b}-1} \frac{d T}{d \xi}\right|_{\xi=\xi_{r}^{-}}
$$


$\Rightarrow$

$$
\begin{aligned}
& \Lambda_{0}= \begin{cases}\frac{D}{\left(T_{b}-1\right) r(\hat{l}-l)} \ln \left[\frac{\hat{l}}{l+(\hat{l}-l) \alpha_{s}}\right], & l \neq \hat{l} \\
\frac{D}{\left(T_{b}-1\right) r l}\left(1-\alpha_{s}\right), & l=\hat{l} .\end{cases} \\
& \theta_{1}\left(\alpha_{0}\right)= \begin{cases}\ln \left(\frac{\ln \left[l+(\hat{l}-l) \alpha_{0}\right]-\ln \left[l+(\hat{l}-l) \alpha_{s}\right]}{\ln \hat{l}-\ln \left[l+(\hat{l}-l) \alpha_{s}\right]}\right), & \hat{l} \neq l \\
\ln \left(\frac{\alpha_{0}-\alpha_{s}}{1-\alpha_{s}}\right), & \hat{l}=l\end{cases} \\
& \Lambda_{0} \Rightarrow \text { Deflagration Speed } \tilde{U}^{2} \sim \frac{\tilde{A} \tilde{\rho}_{l} \tilde{R}^{\circ} \tilde{T}_{b}^{2} e^{-\tilde{E}_{l} / \tilde{R}^{\circ} \tilde{T}_{b}}}{\tilde{D}(\tilde{Q}) \tilde{\rho}_{s} \tilde{E}_{l}} \cdot \tilde{f}\left(\tilde{\lambda}_{g}, \tilde{\lambda}_{l}\right) \\
& \text { where } \frac{\tilde{f}}{\tilde{\lambda}_{l}}= \begin{cases}\frac{\left(\tilde{\lambda}_{g} / \tilde{\lambda}_{l}\right)-1}{\ln \left(\tilde{\lambda}_{g} / \lambda_{l}\right)-\ln \left\{1+\left[\left(\tilde{\lambda}_{g} / \tilde{\lambda}_{l}\right)-1\right] \alpha_{s}\right\}}, & \tilde{\lambda}_{g} \neq \tilde{\lambda}_{l} \\
1 /\left(1-\alpha_{s}\right), & \tilde{\lambda}_{g}=\tilde{\lambda}_{l}\end{cases}
\end{aligned}
$$


Deflagration Speed: $\tilde{U}^{2} \sim \frac{\tilde{A} \tilde{\rho}_{l} \tilde{R}^{\circ} \tilde{T}_{b}^{2} e^{-\tilde{E}_{l} / \tilde{R}^{\circ} \tilde{T}_{b}}}{\tilde{D}(\tilde{Q}) \tilde{\rho}_{s} \tilde{E}_{l}} \cdot \tilde{f}\left(\tilde{\lambda}_{g}, \tilde{\lambda}_{l}\right)$

where $\frac{\tilde{f}}{\tilde{\lambda}_{l}}= \begin{cases}\frac{\left(\tilde{\lambda}_{g} / \tilde{\lambda}_{l}\right)-1}{\ln \left(\tilde{\lambda}_{g} / \lambda_{l}\right)-\ln \left\{1+\left[\left(\tilde{\lambda}_{g} / \tilde{\lambda}_{l}\right)-1\right] \alpha_{s}\right\}}, & \tilde{\lambda}_{g} \neq \tilde{\lambda}_{l} \\ 1 /\left(1-\alpha_{s}\right), & \tilde{\lambda}_{g}=\tilde{\lambda}_{l}\end{cases}$

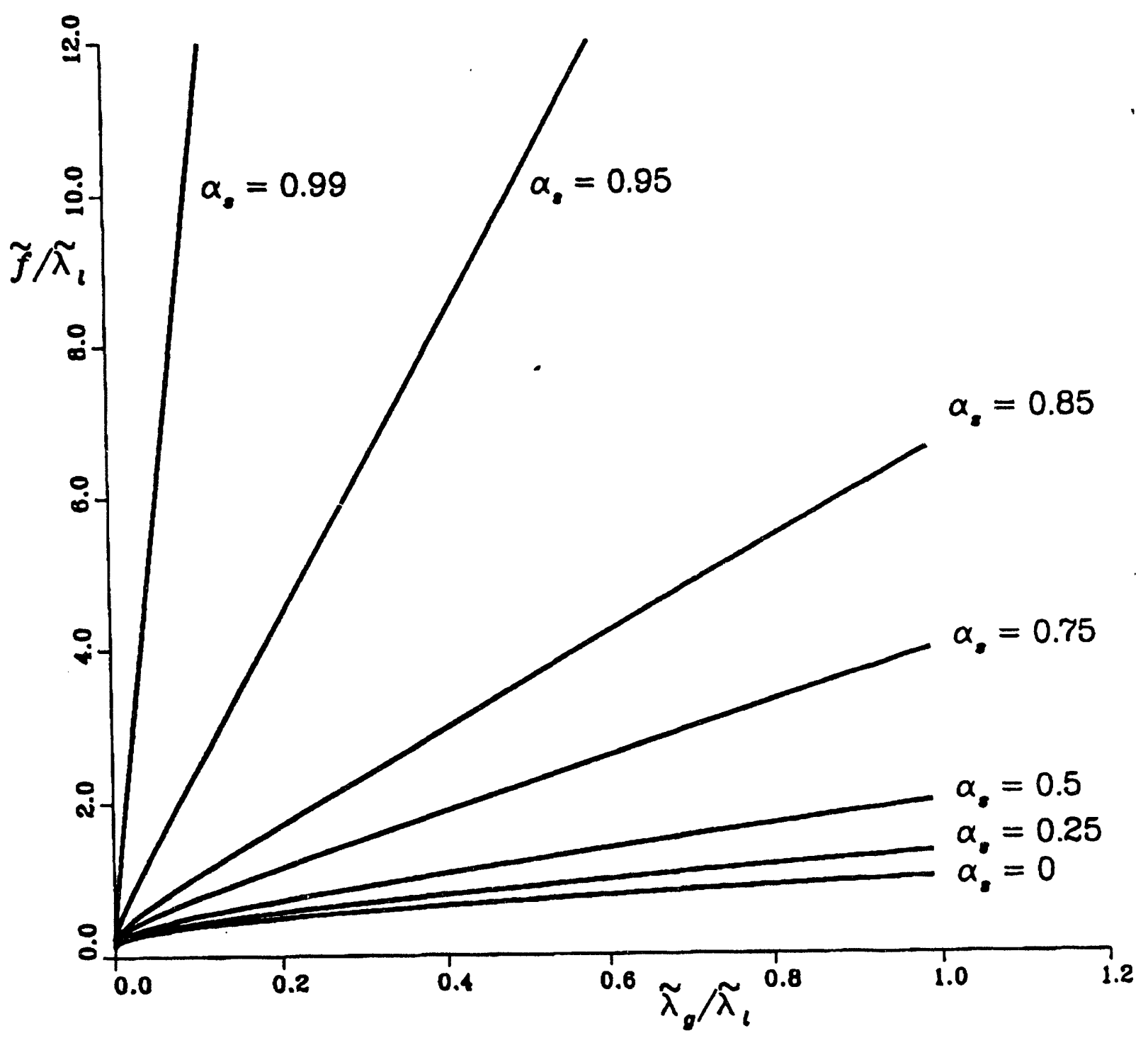


Some General Conclusions on the Deflagration Speed

1. $\tilde{U}$ increases as the thermal conductivity of either phase increases

$$
\left(\partial \tilde{f} / \partial \tilde{\lambda}_{g}>0, \quad \partial \tilde{f} / \partial \tilde{\lambda}_{l}>0\right)
$$

2. $\tilde{U}$ more sensitive to the conductivity of the more thermally conductive phase

3. To leading order in $\beta^{-1}, \tilde{U}$ is independent of the thermal conductivity of the solid

4. $\tilde{U}$ is exponentially sensitive to the burned temperature, which decreases with increasing porosity and pressure 
Results from Two-Temperature Analysis:

Consider regime of large, but finite $K_{l g}, K_{s g}$ :

$$
K_{l g}=\beta^{2} k \Rightarrow \tilde{U}^{-2} \propto \Lambda_{0} \sim \mu+k^{-1} \mu_{1}+k^{-2} \mu_{2}+\cdots,
$$

Zel'dovich Number $\beta \equiv\left(1-T_{b}^{-1}\right) N$

1. First effects of finite rates of interphase heat transfer felt in the liquid/gas reaction zone

2. Resistance to interphase heat transfer $\Rightarrow$ implies that $T_{l}$ generally exceeds $T_{g}$ since heat of reaction is initially deposited in the liquid phase:

$$
\begin{gathered}
T_{l}-T_{g} \sim \frac{\mu\left(T_{b}-1\right)}{\beta k b}(1-\alpha) e^{\theta(\alpha)}\left\{q_{2}-q_{1} \frac{l(1-\alpha)}{l+(\hat{l}-l) \alpha}\left[2+\frac{(\hat{l}-l)(1-\alpha)}{l+(\hat{l}-l) \alpha}\right]\right\}, \\
q_{1}=\frac{(b-\hat{b}) T_{b}+Q}{T_{b}-1}, \quad q_{2}=\frac{Q+b T_{b}}{T_{b}-1}
\end{gathered}
$$

3. As $K_{l g}$ decreases, burning velocity increases due to excess temperature of the reactive liquid phase:

$$
\mu_{1}=-\mu \frac{q_{1}\left(1-\alpha_{s}\right)^{2}}{6 r b l}\left[q_{2}-\frac{1}{5} q_{1}\left(1-\alpha_{s}\right)\left(4+\alpha_{s}\right)\right], \quad(\hat{l}=l)
$$




\section{STABILITY RESULTS $\left(\alpha_{s}=0, \rho_{s}=\right.$ const.)}

Extend asymptotic model to time-dependent, 3D case:

a. weak nonplanarity $\Rightarrow \mathbf{u}_{\mathbf{g}, 1}=\left(0,0, u_{g, l}\right)$

b $u_{g, l}=u_{g, l}\left(x_{1}, x_{2}, t\right)$

c. reaction zone: $x_{3} \sim \Phi_{r}\left(x_{1}, x_{2}, t\right)$

d. nondimensional liquid, gas temperatures $\Theta\left(x_{1}, x_{2}, x_{3}, t\right), \Psi\left(x_{1}, x_{2}, t\right)$ (neglect $\gamma_{s}$, distinction between solid and liquid phases)

\section{Asymptotics: $N \gg 1$}

a. outer zones (preheat, burned regions)

1. exponentially small reaction rate

2. $\alpha=0$ (preheat region) or $\alpha=1$ (burned gas)

b. inner reaction zone (asymptotically thin)

1. quasi-steady, quasi-planar

2. $\left|T_{b}-T_{g, l}\right| \ll 1 \quad\left(K_{l g} \gg 1\right)$

c. matching of inner and outer solutions:

1. Closed Outer Problem with

2. Nonlinear Jump Conditions across thin reaction zone (sheet) 
The Asymptotic Model:

$$
l=\tilde{\lambda}_{g} / \tilde{\lambda}_{l}, \quad b=\tilde{c}_{g} / \tilde{c}_{l}, \quad r=\tilde{\rho}_{g} / \tilde{\rho}_{l}
$$

In terms of $x=x_{1}, y=x_{2}, z=x_{3}-\Phi\left(x_{1}, x_{2}, t\right)$,

$$
\begin{gathered}
\frac{\partial \Theta}{\partial t}+\left(1-\Phi_{t}\right) \frac{\partial \Theta}{\partial z}=\nabla^{2} \Theta, \quad z<0, \\
\frac{\partial \Psi}{\partial t}+\frac{1}{r}\left(1-\Phi_{t}\right) \frac{\partial \Theta}{\partial z}=\frac{l}{b r} \nabla^{2} \Psi, \quad z>0
\end{gathered}
$$

Boundary Conditions:

$$
\begin{aligned}
& \Theta \rightarrow 0 \text { as } z \rightarrow-\infty \\
& \Psi \rightarrow 1 \text { as } z \rightarrow+\infty
\end{aligned}
$$

Jump Conditions:

$$
\begin{aligned}
\Theta & \left.\Theta\right|_{z=0^{-}}=\left.\Psi\right|_{z=0^{+}} \\
\left.l \frac{\partial \psi}{\partial z}\right|_{z=0^{+}}-\left.\frac{\partial \Theta}{\partial z}\right|_{z=0^{-}}= & \frac{\Phi_{t}-1}{1+\Phi_{x}^{2}+\Phi_{y}^{2}}\left[\left.(1-b) \Psi\right|_{z=0^{+}}+b\right] \\
& +(1-l)\left(\left.\Phi_{x} \frac{\partial \Psi}{\partial x}\right|_{z=0^{+}}+\left.\Phi_{y} \frac{\partial \Psi}{\partial y}\right|_{z=0+}\right)
\end{aligned}
$$

Relation for $\Phi_{t}$; e.g.,

$$
\left(\Phi_{t}-1\right)^{2}=\left(1+\Phi_{x}^{2}+\Phi_{y}^{2}\right) \exp \left[\beta\left(\left.\Psi\right|_{z=0^{+}}-1\right)\right] \quad(s=0, K=\infty)
$$


Basic Solution for Steady, Planar Burning:

$$
\Phi_{0}=0 ; \quad \Theta_{0}=e^{z}, \quad z<0 ; \quad \Psi_{0}=1, \quad z>0
$$

Linear Stability Analysis:

$$
\Phi=\varphi, \quad \Theta=e^{z}+\vartheta+\varphi e^{z}, \quad \Psi=1+\varsigma
$$

Substituting into asymptotic model and linearizing $\Rightarrow$

$$
\begin{gathered}
\left\{\begin{array}{c}
\varphi \\
\vartheta \\
\varsigma
\end{array}\right\}=e^{i \omega t \pm i k_{1} x \pm i k_{2} y}\left\{\begin{array}{c}
1 \\
c_{1} e^{p z} \\
c_{2} e^{q z}
\end{array}\right\}, \quad k=\sqrt{k_{1}^{2}+k_{2}^{2}}, \\
p=\frac{1}{2}\left[1+\sqrt{1+4\left(i \omega+k^{2}\right)}\right], \quad q=\frac{1}{2 l}\left[b-\sqrt{b^{2}+4\left(i \omega r b l+l^{2} k^{2}\right)}\right] \\
+ \text { dispersion relation for } \omega(k)
\end{gathered}
$$

Linear Stability Determined by $\omega(k)$ :

a. neutral stability $\Rightarrow \operatorname{Re}(i \omega)=0$

b. $\operatorname{Im}(i \omega) \neq 0$ on neutral stability boundary

$$
\Rightarrow \text { Intrinsic Oscillatory Stability Boundary } \beta(k)
$$

Related to well-known condensed-phase combustion instability for

a. SHS (combustion synthesis); cf. Makhviladze and Novozhilov (1971), Margolis $(1983,1985)$

b. nonporous solid propellants without two-phase flow; cf. Denison and Baum (1961), Margolis and Williams (1988) 


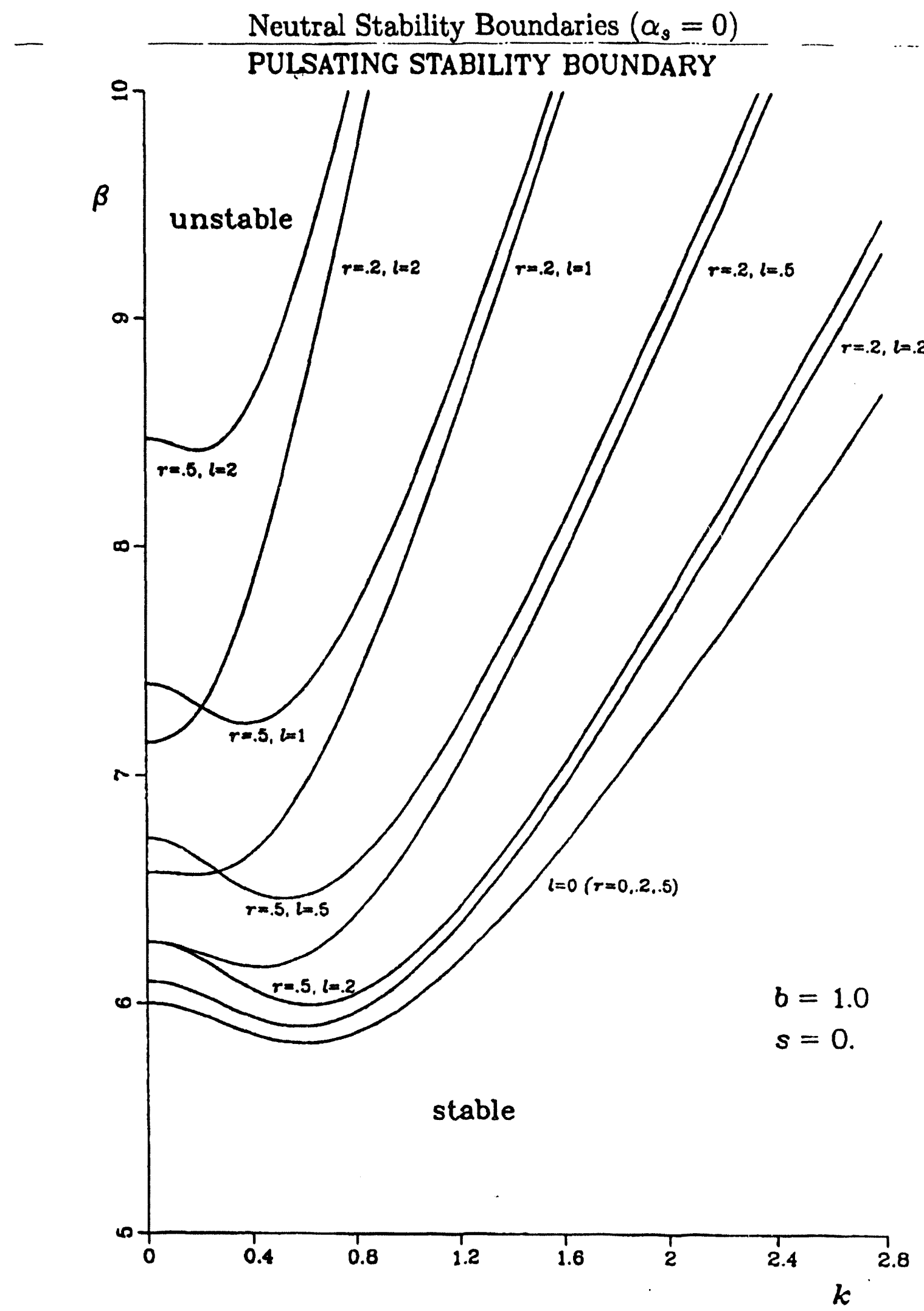

Neutral stability boundaries in the limit $K=\infty$ and $s=0$, for various values of the remaining parmaters. Decreasing values of $r$ and $l$ are destabilizing. 


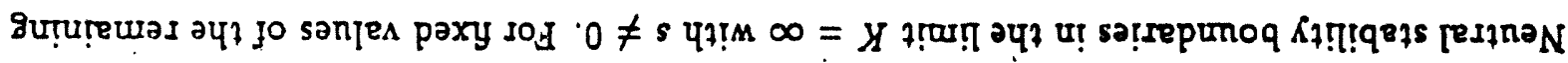

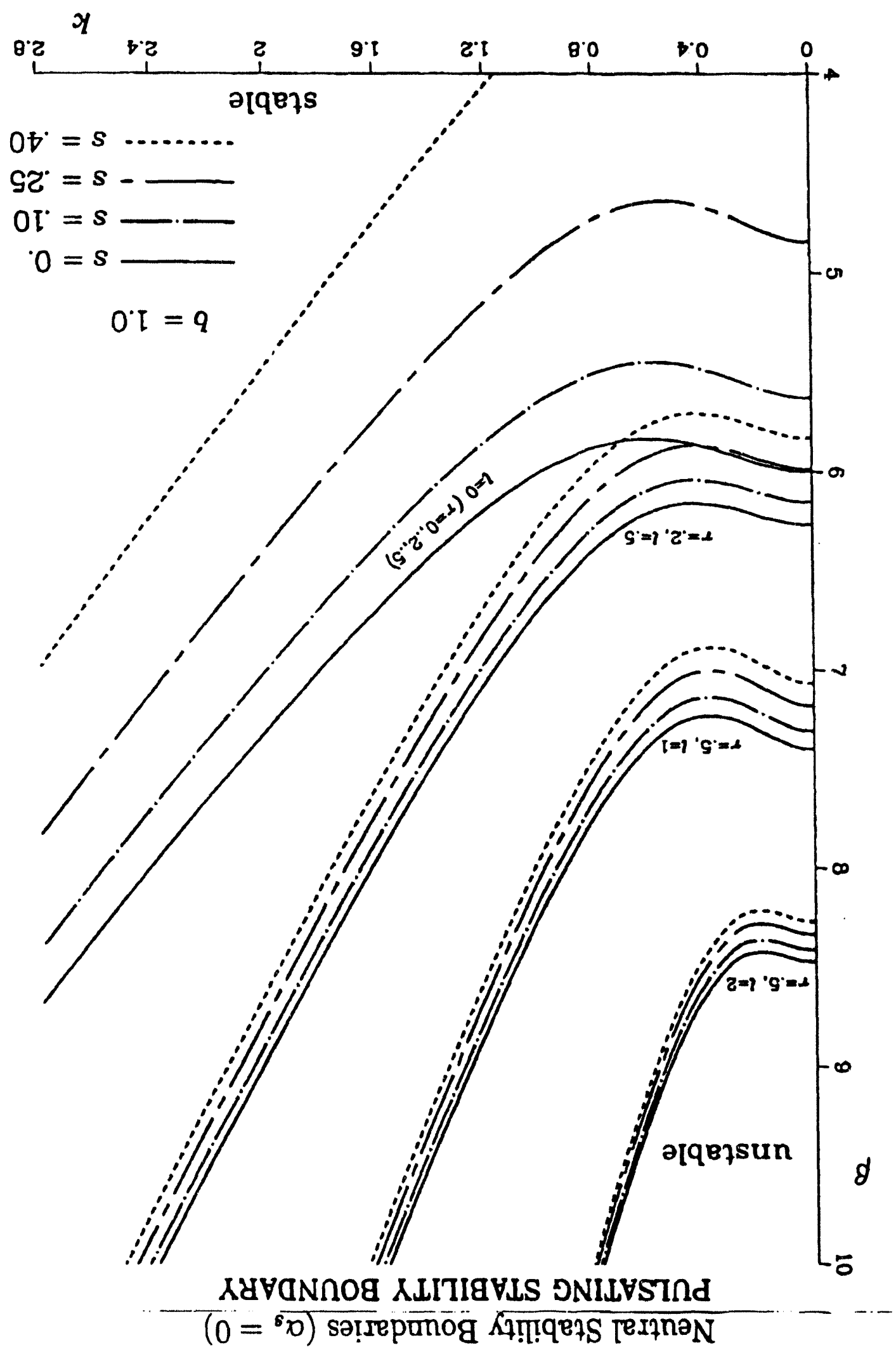


Neutral Stability Boundaries $\left(\alpha_{s}=0\right)$

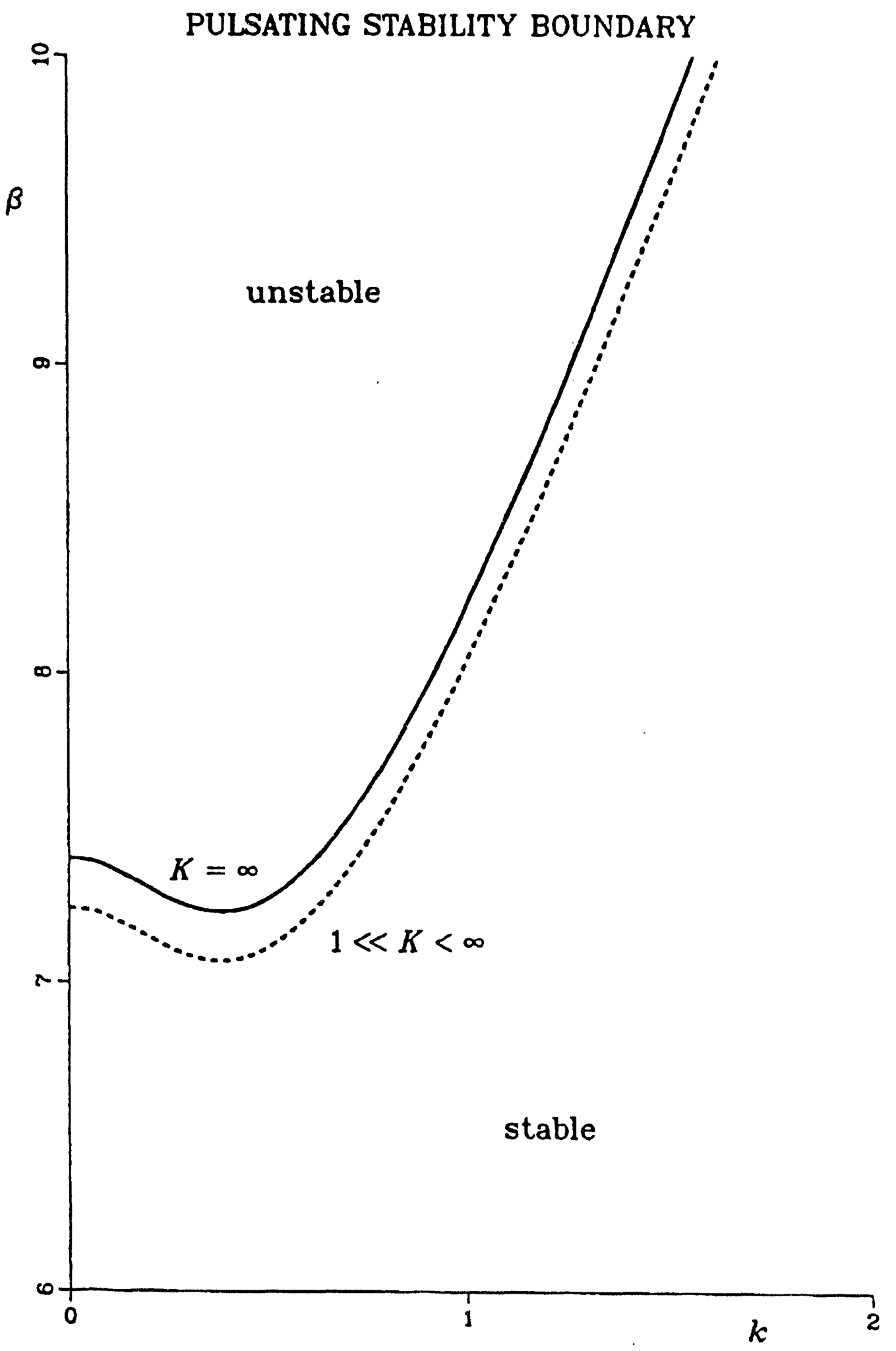

Neutral stability boundaries for $K=\infty$ and $1 \ll K<\infty$, showing that finite values of $K$ are destabilizing. 


\section{Stability Conclusions}

1. Increasing values of $r$ and $l$ are stabilizing (gas has stabilizing effect on what is essentially a condensed-phase instability)

2. Decreasing values of $K_{l g}$ are destabilizing (less thermal coupling between gas and liquid reduces gas-phase damping)

3. Increasing values of velocity-perturbation (Marangoni) parameter $s$ is destabilizing (increases discrepancy between liquid- and gas-phase velocities) 

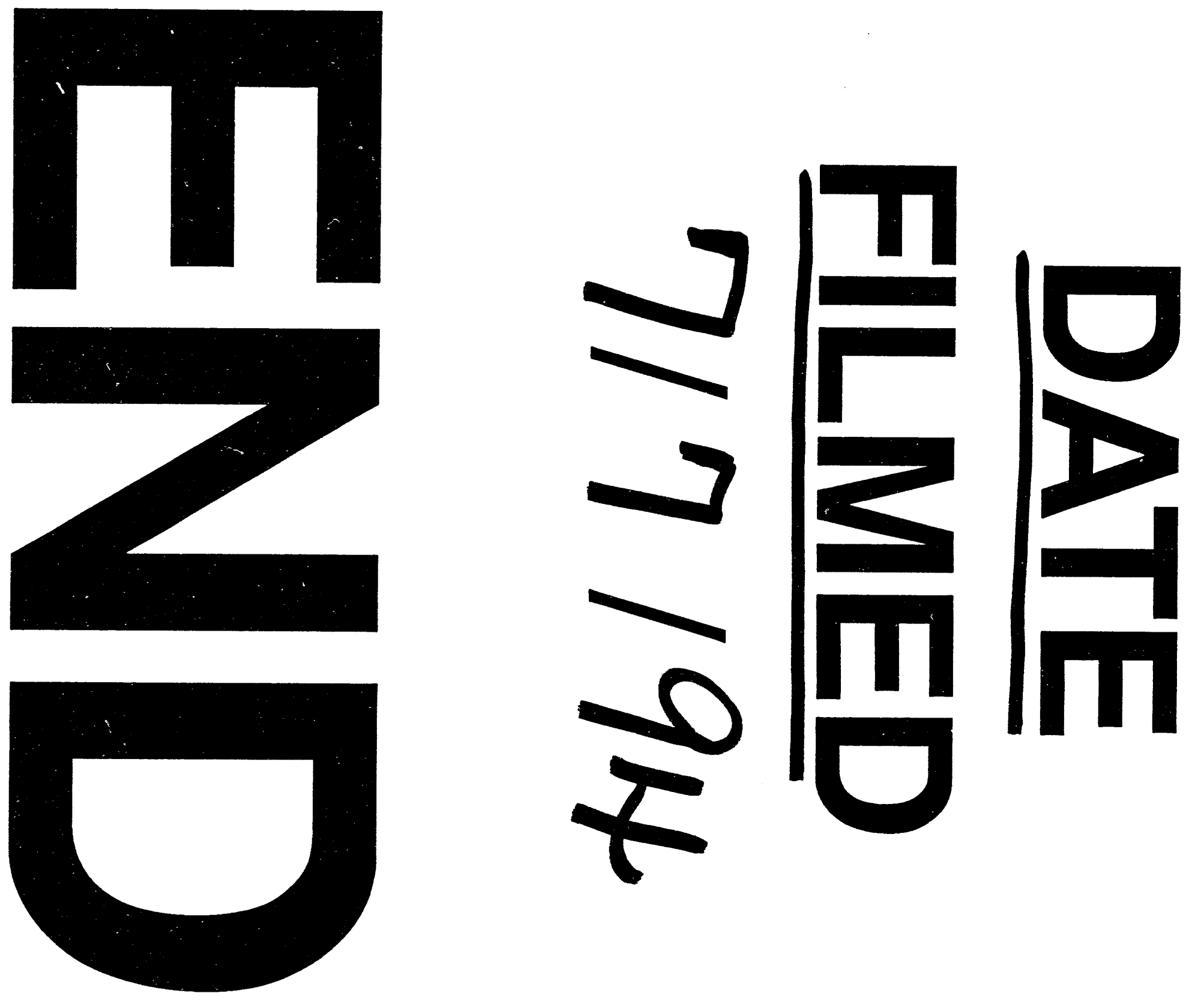
